\title{
Study of InN surface by high resolution electron energy loss spectroscopy (HREELS)
}

\author{
Ananta R. Acharya and Brian D. Thoms \\ Georgia State University, Department of Physics and Astronomy, Atlanta, GA 30303 \\ acharyaananta@yahoo.com
}

\begin{abstract}
Understanding the surface structure and termination of semiconductor thin films during epitaxial growth is critical since it affects the structural, interfacial and electronic properties of epilayers and device structures. The structural properties and surface bonding configuration of InN layers grown by high pressure chemical vapor deposition (HPCVD) have been characterized using high resolution electron energy loss spectroscopy (HREELS). The HREELS analysis of these InN layers shows that the surface is predominantly terminated with $\mathrm{NH}_{2}$ species. This is in contrast to previous work on HPCVD-grown InN layers, which showed only NH species on the nitrogen terminated surface. The presence of $\mathrm{NH}_{2}$ related modes of vibration is the indication of the orientation of the surface other than c-plane.
\end{abstract}

Key words: HREELS, Indium nitride, $\mathrm{NH}_{2}$

\section{INTRODUCTION}

InN, one of the group III nitride semiconductors, has been an interesting area of research due to its inherent properties which can increase the performance of photovoltaics, electronics and optoelectronics devices. It is a direct and narrow band gap (0.65 eV) semiconductor. Among all III-nitride semiconductors, InN has the lowest effective mass for electrons ${ }^{1}$ which leads to high mobility and high saturation velocity. Using InN and its alloys with GaN and AIN, emission of nitride-based LEDs can be extended from ultraviolet to near infrared region. For example, InGaN, a ternary alloy of $\mathrm{InN}$ and $\mathrm{GaN}$, has been found very useful in optoelectronic devices, such as, LEDs $^{2}$ and lasers. ${ }^{3}$ By changing the In content, the band gap of InGaN can be tuned over a wide range corresponding to wavelengths from infrared to ultraviolet region. This important property of InGaN alloy has made possible to fabricate high efficiency photovoltaic cells capturing most of the solar spectrum. ${ }^{4}$

Despite the enticing properties for device fabrication, the growth of high crystalline quality $\mathrm{InN}$ remains a challenge, especially due to two reasons: stoichiometric instability and low dissociation temperature. Using low pressure chemical vapor deposition (LPCVD) at low temperatures about $600{ }^{\circ} \mathrm{C}$, thermal desorption is suppressed..$^{5}$ However, the cracking efficiency of ammonia in this temperature regime is extremely low and it requires a large group V-III precursor ratio. ${ }^{6}$ Even at high temperature regime, the growth of highly volatile compounds, such as InN, is possible if surface stabilization is done at high pressures. High pressure chemical vapor deposition (HPCVD) has been found as an effective technique to grow stoichimetric $\mathrm{InN}$ layers at elevated temperatures $\left(\sim 900^{\circ} \mathrm{C}\right) .^{7}$

HREELS is a surface sensitive vibrational spectroscopic technique in which low energy electrons are incident on the surface of the sample. The scattered electrons are collected in a specular direction and energy is analysed. Electrons with energy loss up to $500 \mathrm{meV}$ are counted to accumulate a spectrum. Our HREELS spectrometer consists of a cathode made of lanthanum hexaboride and a pair of cylindrical monochromators and analyzers. A symmetrical system of input-output zoom lenses are positioned between the monochromators and analyzers in order to focus, accelerate or decelerate beam of electrons to and from the sample surface. HREELS experiments were performed in a stainless-steel ultrahigh vacuum (UHV) system with base pressure of $2 \times 10^{-}$ 10 Torr. The UHV system is also equipped with Auger electron spectroscopy (AES), low energy electron diffraction (LEED) and ion gun apparatus. The chamber is pumped by three pumps; two turbo molecular pumps and one ion pump. The need of this much low pressure is mainly to keep the surface clean and avoid any kind of collisions or interactions with air molecules. The details of the UHV system and sample mount have already been reported by Bellitto et al. ${ }^{8}$ 


\section{EXPERIMENTAL METHODS}

The InN layers, characterized here, were grown by HPCVD process in Dr. Dietz's lab at Georgia State University in the department of Physics and Astronomy. The sample was grown on a sapphire substrate at a temperature of $865{ }^{\circ} \mathrm{C}$, a reactor pressure of $15 \mathrm{bar}$, and an ammonia $\left(\mathrm{NH}_{3}\right)$ to trimethylindium (TMI) precursor ratio of 3000 . The sample was cleaned with acetone and rinsed with isopropyl alcohol before installing into the UHV chamber. The ultrahigh vacuum condition was restored in the chamber by a 5 day long baking and cooling method. After maintaining the UHV environment in the chamber, the sample was cleaned by a procedure of bombardment with $1 \mathrm{keV}$ nitrogen ions followed by atomic hydrogen cleaning (AHC), which was reported as an effective technique to remove surface contaminants by Piper et al. ${ }^{9} \mathrm{AHC}$ was performed by backfilling the vacuum chamber with hydrogen to a pressure of $8.4 \times 10^{-7}$ Torr in the presence of a tungsten filament heated to $1575{ }^{\circ} \mathrm{C}$ to produce $\mathrm{H}$-atoms. The sample was kept at 20 $\mathrm{mm}$ from the dosing filament for 5 minutes (giving an exposure of $250 \mathrm{~L}$ of hydrogen, $1 \mathrm{~L}=1 \times 10^{-6}$ Torr s). After 5 minutes, the sample was heated to $350^{\circ} \mathrm{C}$ by the bombardment of the electrons from the back of the sample mount for 10 minutes keeping the exposure of $\mathrm{H}$-atom same as before (giving an additional exposure of $500 \mathrm{~L}$ of hydrogen). In order to monitor the surface cleanliness, Auger electron spectroscopy (AES) was used. The procedure of cleaning was repeated until the surface of the sample was free from contaminants as confirmed by AES. After the sample was found in good shape, HREELS experiments were performed in a specular geometry with an incident and scattered angle of $60^{\circ}$ from the surface normal, and an incident electron beam energy of 5.6 $\mathrm{eV}$.

\section{RESULTS AND DISCUSSION}

Fig. 1 shows the HREELS spectra of InN epilayers grown on GaN/sapphire studied by authors' research group previously. ${ }^{10}$ The strong loss feature at $550 \mathrm{~cm}^{-1}$ was assigned to a Fuchs- Kliewer surface phonon. The loss peak at $3260 \mathrm{~cm}^{-1}$ for the atomic hydrogen cleaned (AHC) surface was assigned to an N-H stretching vibration, while the loss feature at $870 \mathrm{~cm}^{-1}$ was assigned to an $\mathrm{N}-\mathrm{H}$ bending vibration. In addition, the peak near $360 \mathrm{~cm}^{-1}$ was suggested to the bouncing vibrations of the nitrogen surface atoms with the surface. From these observations it was concluded that the $\mathrm{InN}$ film in that work was N-polar and N-H terminated.

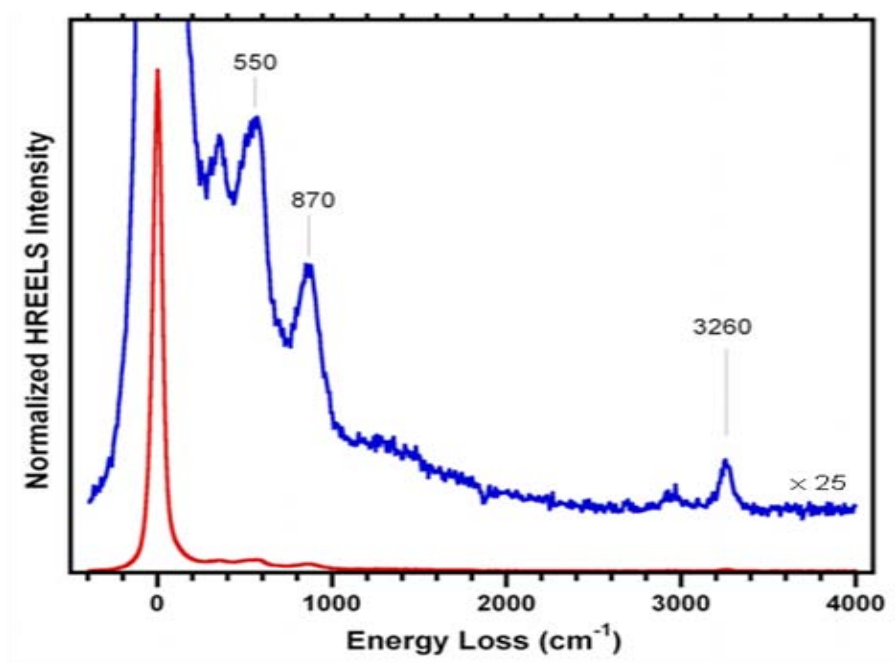

Fig. 1. HREELS of InN after AHC. Spectra were acquired in the specular direction with an incident electron energy of $12.5 \mathrm{eV}$.

In another work, ${ }^{11}$ HREELS spectra of the AHC treated InN layers were observed as shown in Fig. 2. In this work, they varied the energy of the incident electrons and observed a strong loss feature at $560 \mathrm{~cm}^{-1}$ in all spectra. This loss feature was assigned to a Fuchs-Kliewer surface phonon. Most importantly, they observed a broad loss feature in the range of $3100-3600 \mathrm{~cm}^{-1}$ for different incident electron energies which they assigned to a conduction band plasmon excitation. From these spectra they revealed the fact of the presence of a surface electron accumulation.

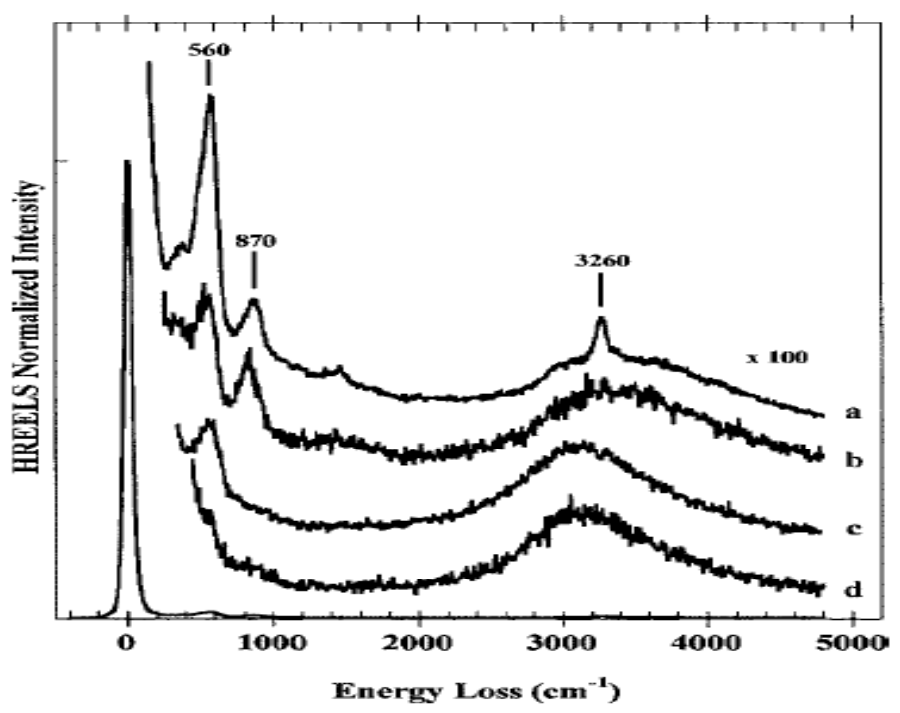

Fig. 2. HREELS from an atomic hydrogen cleaned InN sample with incident electron energies of (a) $7 \mathrm{eV}$, (b) $15 \mathrm{eV}$, (c) $25 \mathrm{eV}$, and (d) $35 \mathrm{eV}$. 
In the present work, the HREELS spectrum of an InN sample obtained after AHC is depicted in Fig. 3. As shown in Fig. 3, loss features are observed at $640 \mathrm{~cm}^{-1}, 1260 \mathrm{~cm}^{-1}, 1440 \mathrm{~cm}^{-1}, 1600 \mathrm{~cm}^{-1}$, $2960 \mathrm{~cm}^{-1}, 3400 \mathrm{~cm}^{-1}$ and $3710 \mathrm{~cm}^{-1}$. The loss peaks at 1260, 1600 and $3400 \mathrm{~cm}^{-1}$ indicate that this surface is predominantly $\mathrm{NH}_{2}$ terminated as opposed to the $\mathrm{N}-\mathrm{H}$ termination observed in earlier work. ${ }^{10-11}$ Unlike in the previous work $^{10}$ where they observed Fuchs-Kliewer surface phonon at $550 \mathrm{~cm}^{-1}$, a strong and broad loss feature is observed at $640 \mathrm{~cm}^{-1}$ in the present work. Tindall et al. ${ }^{12}$ reported a rocking mode of $\mathrm{NH}_{2}$ vibration at $884 \mathrm{~cm}^{-1}$ on $\mathrm{Ge}(100)$ surface. The strong and broad loss feature at $640 \mathrm{~cm}^{-1}$ is assigned to the unresolved overlap of the Fuchs-Kliewer surface phonon and the rocking mode of the $\mathrm{NH}_{2}$ vibration. The loss feature at $3400 \mathrm{~cm}^{-1}$ is assigned to an $\mathrm{NH}_{2}$ stretching vibration in agreement with that assigned by Tanaka et al. ${ }^{13}$ for $\mathrm{NH}_{2}$ symmetric stretching vibration on $\operatorname{InP}(110)$. The loss features at $1260 \mathrm{~cm}^{-1}$ and at 1600 $\mathrm{cm}^{-1}$ are assigned to $\mathrm{NH}_{2}$ wagging and scissor modes of vibration respectively which have close agreement with those assigned by Tindall et al. ${ }^{12}$ on $\mathrm{Ge}(100)$.

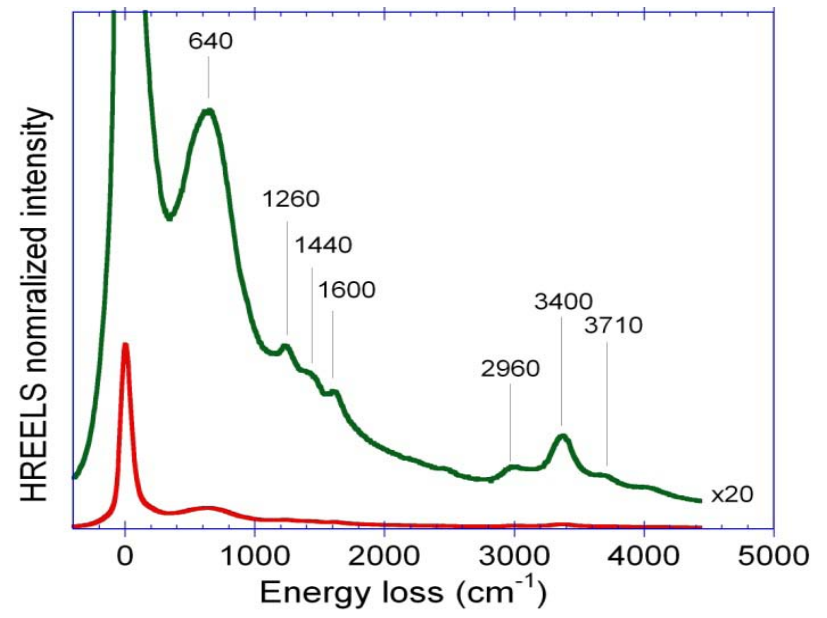

Fig. 3. HREEL spectra of HPCVD-grown InN after atomic hydrogen cleaning.

Small loss features at $1440 \mathrm{~cm}^{-1}$ and at $2960 \mathrm{~cm}^{-1}$ have been assigned to the bending and stretching modes of $\mathrm{C}-\mathrm{H}$ vibrations respectively. A small loss feature at $3710 \mathrm{~cm}^{-1}$ is assigned to stretch mode of $\mathrm{O}-\mathrm{H}$ vibration. The loss feature at $3400 \mathrm{~cm}^{-1}$ is assigned to an $\mathrm{NH}_{2}$ stretching vibration in agreement with that assigned by Tanaka et al. ${ }^{13}$ for $\mathrm{NH}_{2}$ symmetric stretching vibration on $\operatorname{InP}(110)$. Nienhaus et al. ${ }^{14}$ reported $\mathrm{InH}$ stretching vibration at $1650-1700$ $\mathrm{cm}^{-1}$ in HREELS experiments and at $1630-1650 \mathrm{~cm}^{-1}$ from ab initio calculations ${ }^{15}$ on InP surfaces. However, no loss feature in this range was observed in the present work indicating no surface $\mathrm{InH}$, i.e. no metallic indium on the surface. In the HREEL spectrum, the appearance of the loss features related to $\mathrm{NH}_{2}$ species implies that the surface is predominantly N-terminated. Since no evidence of metallic indium is observed in our HREELS spectrum, it can be concluded that the presence of $\mathrm{NH}_{2}$ species on InN layer is due to the tilted facets.

\section{CONCLUSION}

In summary, InN surface grown by HPCVD has been characterized using HREELS. The termination of the surface depends upon the growth parameters and the type of the substrate. The HREEL spectrum shows that the $\mathrm{InN}$ surface is mainly $\mathrm{NH}_{2}$ terminated. The presence of $\mathrm{NH}_{2}$ bonds at the InN surface is directly related to tilted $\mathrm{InN}$ facets and is not observed on the c-plane $\mathrm{InN}$ surface.

\section{References}

1. S.N. Mohammad and H. Morkoc, 1996. Prog. Quantum Electron 20, 361.

2. I. Akasaki, H. Amano, N. Koide, M. Kotaki, and K. Manabe, 1993. Physica B 185, 428.

3. S. Nakamura, M. Senoh, S. Nagahama, N. Iwasa, T. Yamada, T. Matsushita, H. Kiyoku, and Y. Sugimoto,1994. Jpn. J. Appl. Phys. 75, 7365.

4. Akio Yammoto, Md. R. Islam, Ting-Ting Kang, and Akihiro Hashimoto, 2010. Phys. Stat. Sol. (c), 1-8 (2010).

5. A.G Bhuiyan, A. Hashimoto, and A. Yamamoto, 2003. J. Appl. Phys. 94 , p.2779-2808.

6. N. Dietz, M. Strassburg, and V. Woods,2005. J. Vac. Sci. Technol. A 23, 1221.

7. N. Dietz, in III-Nitrides Semiconductor Materials, edited by Z. C. Feng Imperial College Press, pp. 203255 (2006).

$8 . \quad$ V. J. Bellitto, B. D. Thoms, D. D. Koleske, A. E. Wickenden, R. L. Henry, Surf. Sci. 430, 80 (1999).

9. L. F. J. Piper, T. D. Veal, M. Walker, I. Mahboob, C. F. McConville, H. Lu, and W. J. Schaff, J. Vac. Sci. Technol. A 23, 617 (2005).

10. R. P. Bhatta, B. D. Thoms, M. Alevli, V. Woods, and N. Dietz, Appl. Phys. Lett. 88, 122112 (2006).

11. R. P. Bhatta, B. D. Thoms, M. Alevli, and N. Dietz, Surf. Sci. 601, L120 (2007).

12. C. Tindall, and J.C. Hemminger, Surf. Sci. 330, 67 (1995).

13. S. Tanaka, M. Onchi, and M. Nishijima, Surf.Sci.191, L756 (1987).

14. H. Nienhaus, S. P. Grabowski, and W. Monch, Surf. Sci. 368, 196 (1996).

15. J. Fritsch, A. Echert, P. Pavone, and U. Schroder, J. Phys.: Condens. Matter 7, 7717 (1995). 